\title{
Enumeration of alternating sign matrices of even size (quasi)-invariant under a quarter-turn rotation
}

\author{
Jean-Christophe Aval and Philippe Duchon ${ }^{\dagger}$ \\ LaBRI, Université Bordeaux 1, CNRS \\ 351 cours de la Libération, 33405 Talence cedex, FRANCE
}

\begin{abstract}
The aim of this work is to enumerate alternating sign matrices (ASM) that are quasi-invariant under a quarter-turn. The enumeration formula (conjectured by Duchon) involves, as a product of three terms, the number of unrestrited ASm's and the number of half-turn symmetric ASM's.

Résumé. L'objet de ce travail est d'énumérer les matrices à signes alternants (ASM) quasi-invariantes par rotation d'un quart-de-tour. La formule d'énumération, conjecturée par Duchon, fait apparaître trois facteurs, comprenant le nombre d'ASM quelconques et le nombre d'ASM invariantes par demi-tour.
\end{abstract}

\section{Introduction}

An alternating sign matrix is a square matrix with entries in $\{-1,0,1\}$ and such that in any row and column: the non-zero entries alternate in sign, and their sum is equal to 1 . Their enumeration formula was conjectured by Mills, Robbins and Rumsey (5), and proved by Zeilberger (9), and almost simultaneously by Kuperberg (3). Kuperberg used a bijection between the ASM's and the states of the statistical square-ice model, for which he studied and computed the partition function. He also used this method in (4) to obtain many enumeration or equinumeration results for various classes of symmetries of ASM's, most of them having been conjectured by Robbins (7). Among these results can be found the following remarkable one.

Theorem 1 (Kupeberg). The number $A_{\mathrm{QT}}(4 N)$ of ASM's of size $4 N$ invariant under a quarter-turn (QTASM's) is related to the number $A(N)$ of (unrestricted) ASM's of size $N$ and to the number $A_{\mathrm{HT}}(2 N)$ of ASM's of size $2 N$ invariant under a half-turn by the formula:

$$
A_{\mathrm{QT}}(4 N)=A_{\mathrm{HT}}(2 N) A(N)^{2} \text {. }
$$

More recently, Razumov and Stroganov (6) applied Kuperberg's strategy to settle the following result, also conjectured by Robbins (7) and relative to QTASM's of odd size.

Theorem 2 (Razumov, Stroganov). The numbers of QTASM's of odd size are given by the following formulas, where $A_{\mathrm{HT}}(2 N+1)$ is the number of HTASM's of size $2 N+1$ :

$$
\begin{aligned}
& A_{\mathrm{QT}}(4 N-1)=A_{\mathrm{HT}}(2 N-1) A(N)^{2} \\
& A_{\mathrm{QT}}(4 N+1)=A_{\mathrm{HT}}(2 N+1) A(N)^{2} .
\end{aligned}
$$

It is easy to observe (and will be proved in Section 2) that the set of QTASM's of size $4 N+2$ is empty. But, by slightly relaxing the symmetry condition at the center of the matrix, Duchon introduced in (2) the notion of ASM's quasi-invariant under a quarter turn (the definition will be given in Section 2) whose class is non-empty in size $4 N+2$. Moreover, he conjectured for these qQTASM's an enumeration formula that perfectly completes the three previous enumeration results on QTASM. This is the aim of this paper to establish this formula.

\footnotetext{
†This work has been supported by the ANR project MARS (BLAN06-2_0193) 
Theorem 3 The number $A_{\mathrm{QT}}(4 N+2)$ of qQTASM of size $4 N+2$ is given by:

$$
A_{\mathrm{QT}}(4 N+2)=A_{\mathrm{HT}}(2 N+1) A(N) A(N+1) .
$$

This paper is organized as follows: in Section 2, we define qQTASM's; in Section 3, we recall the definitions of square ice models, precise the parameters and the partition functions that we shall study, and give the formula corresponding to equation (4) at the level of partition functions; the Section 4 is devoted to the proofs.

\section{ASM's quasi-invariant under a quarter-turn}

The class of ASM's invariant under a rotation by a quarter-turn (QTASM) is non-empty in size $4 N-1$, $4 N$, and $4 N+1$. But this is not the case in size $4 N+2$.

Lemma 4 There is no QTASM of size $4 N+2$.

Proof: Let us suppose that $M$ is a QTASM of even size $2 L$. Now we use the fact that the size of an ASM is given by the sum of its entries, and the symmetry of $M$ to write:

$$
2 L=\sum_{1 \leq i, j \leq 2 L} M_{i, j}=4 \times \sum_{1 \leq i, j \leq L} M_{i, j}
$$

which implies that the size of $M$ has to be a multiple of 4 .

Duchon introduced in (2) a notion of ASM's quasi-invariant under a quarter-turn, by slightly relaxing the symmetry condition at the center of the matrix. The definition is more simple when considering the height matrix associated to the ASM, but can also been given directly.

Definition 5 An ASM $M$ of size $4 N+2$ is said to be quasi-invariant under a quarter-turn (qQTASM) if its entries satisfy the quarter-turn symmetry

$$
M_{4 N+2-j+1,4 N+2-i+1}=M_{i, j}
$$

except for the four central entries $\left(M_{2 N, 2 N}, M_{2 N, 2 N+}, M_{2 N+1,2 N}, M_{2 N+1,2 N+1}\right)$ that have to be either $(0,-1,-1,0)$ or $(1,0,0,1)$.

We give below two examples of qQTASM's of size 6 , with the two possible patterns at the center.

$$
\left(\begin{array}{cccccc}
0 & 0 & 0 & 1 & 0 & 0 \\
0 & 0 & 1 & 0 & 0 & 0 \\
1 & 0 & 0 & -1 & 1 & 0 \\
0 & 1 & -1 & 0 & 0 & 1 \\
0 & 0 & 0 & 1 & 0 & 0 \\
0 & 0 & 1 & 0 & 0 & 0
\end{array}\right) \quad\left(\begin{array}{cccccc}
0 & 0 & 1 & 0 & 0 & 0 \\
0 & 1 & -1 & 0 & 1 & 0 \\
0 & 0 & 1 & 0 & -1 & 1 \\
1 & -1 & 0 & 1 & 0 & 0 \\
0 & 1 & 0 & -1 & 1 & 0 \\
0 & 0 & 0 & 1 & 0 & 0
\end{array}\right)
$$

In the next section, we associate square ice models to ASM's with various types of symmetry.

\section{Square ice models and partition functions}

\subsection{Notations}

Using Kuperberg's method we introduce square ice models associated to ASM, HTASM and QTASM. We recall here the main definitions and refer to (4) for details and many examples.

Let $a \in \mathbb{C}$ be a global parameter. For $x$ any complex number different from zero, we denote $\bar{x}=1 / x$, and we define:

$$
\sigma(x)=x-\bar{x} .
$$




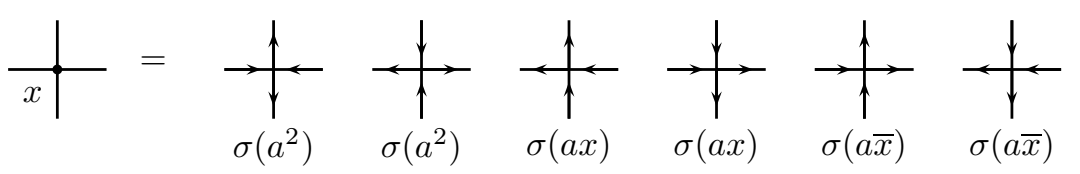

Fig. 1: The 6 possible orientations and associated weights

If $G$ is a tetravalent graph, an ice state of $G$ (we will sometimes call them configurations) is an orientation of the edges such that every tetravalent vertex has exactly two incoming and two outcoming edges.

A parameter $x \neq 0$ is assigned to any tetravalent vertex of the graph $G$. Then this vertex gets a weight, which depends on its orientations, as shown on Figure 1.

It is sometimes easier to assign parameters, not to each vertex of the graph, but to the lines that compose the graph. In this case, the weight of a vertex is defined as:

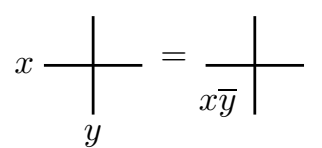

When this convention is used, a parameter explicitly written at a vertex replaces the quotient of the parameters of the lines.

We will put a dashed line to mean that the parameter of a line is different on the sides of the dashed line. We will also use divalent vertices, and in this case the two edges have to be both in, or both out, and the corresponding weight is 1 :

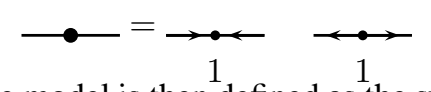

The partition function of a given ice model is then defined as the sum over all its states of the product of the weights of the vertices.

To simplify notations, we will denote by $X_{N}$ the vector of variables $\left(x_{1}, \ldots, x_{N}\right)$. We use the notation $X \backslash x$ to denote the vector $X$ without the variable $x$.

\subsection{Partition functions for classes of ASM's}

We give in Figures 2, 3, and 4 the ice models corresponding to the classes of ASM's that we shall study, and their partition functions. The bijection between ASM's and states of the square ice model with "domain wall boundary" is now well-known ( $c f$. (4)), and the bijections for the other classes of symmetry may be easily checked in the same way.

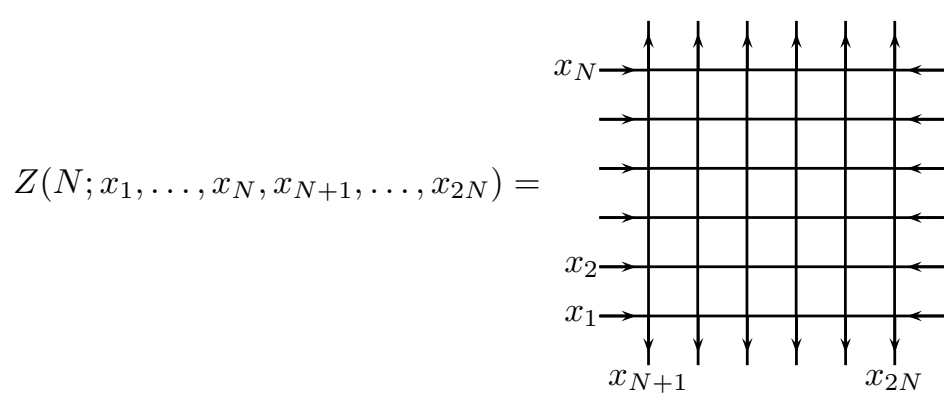

Fig. 2: Partition function for ASM's of size $N$

With these notations, Theorem 3 will be a consequence of the following one which addresses the concerned partition functions. 


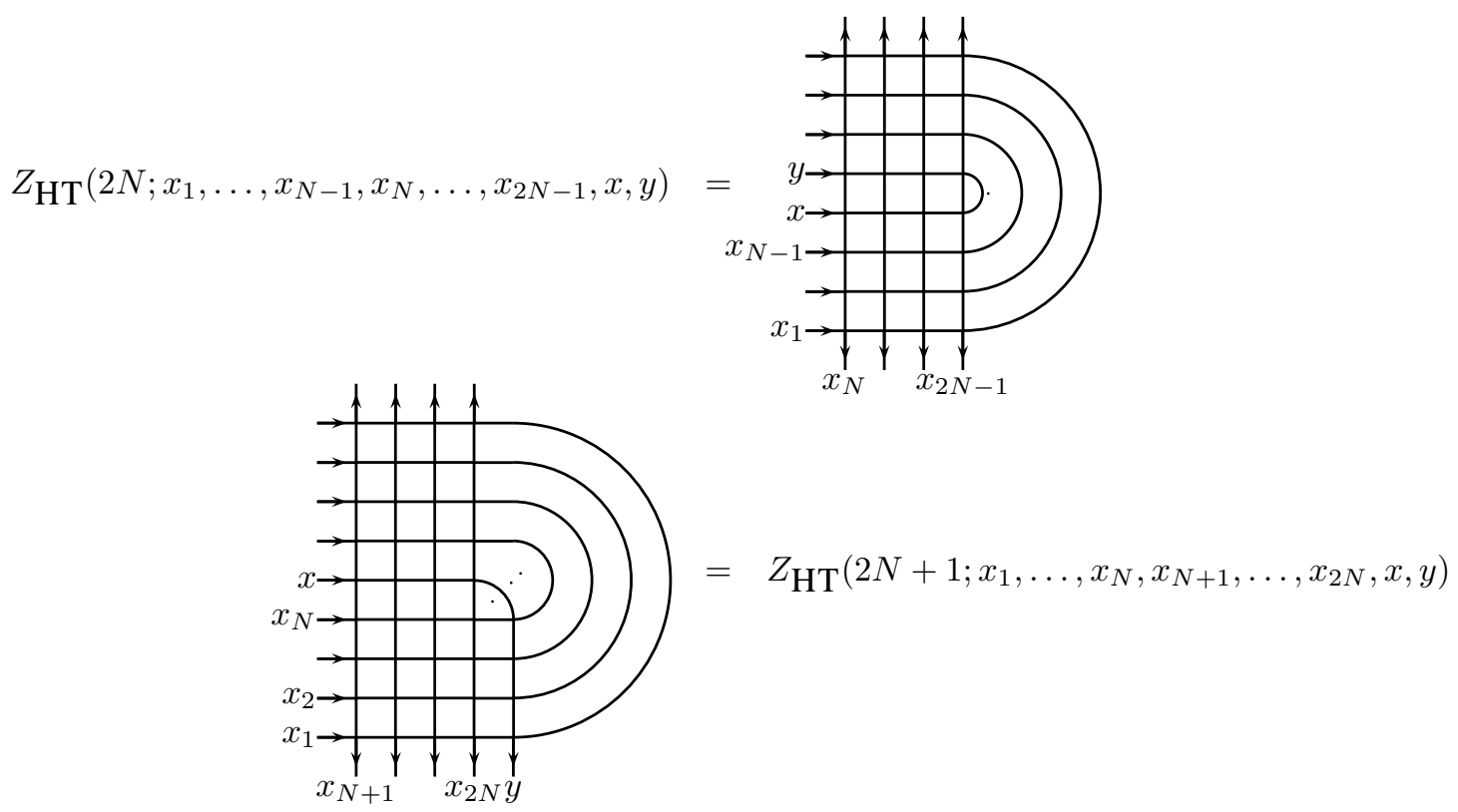

Fig. 3: Partition functions for HTASM's

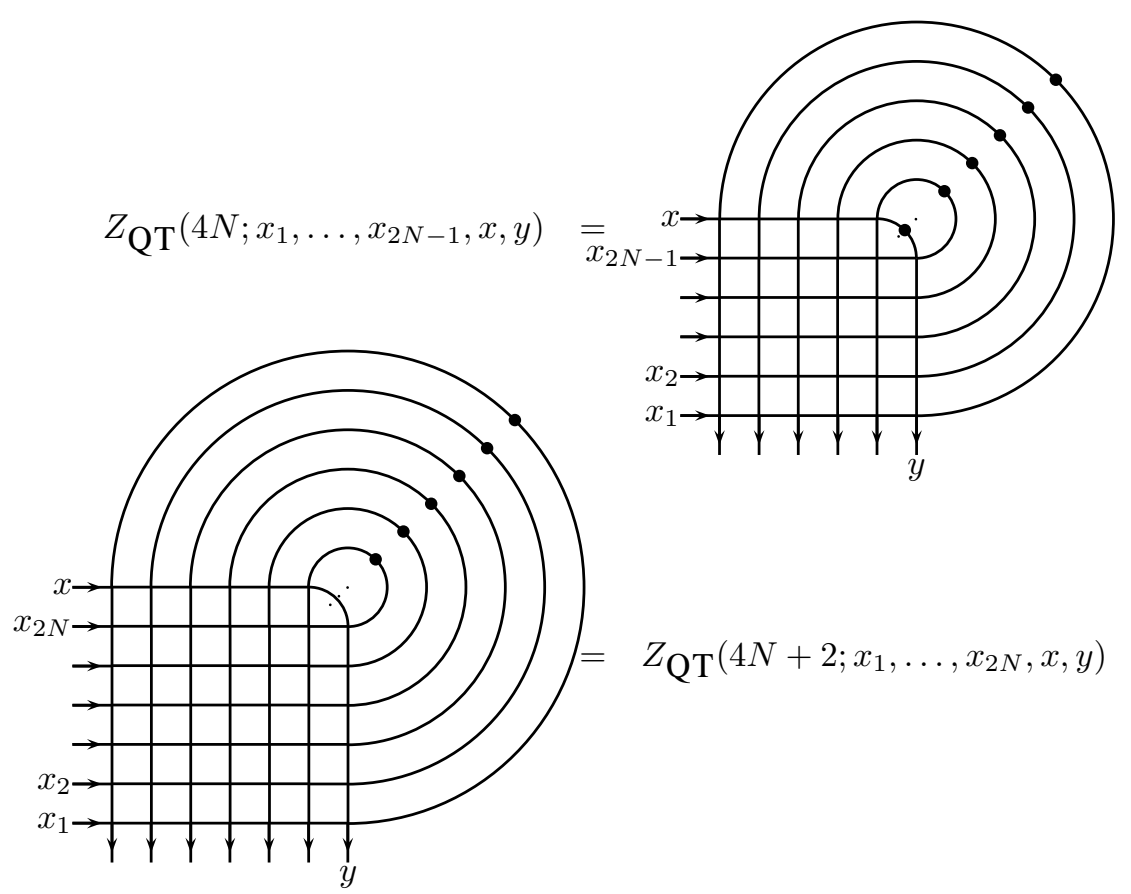

Fig. 4: Partition functions for (q)QTASM of even size 
Theorem 6 When $a=\omega_{6}=\exp (i \pi / 3)$, one has for $N \geq 1$ :

$$
Z_{\mathrm{QT}}\left(4 N ; X_{2 N-1}, x, y\right)=\sigma(a)^{-1} Z_{\mathrm{HT}}\left(2 N ; X_{2 N-1}, x, y\right) Z\left(N ; X_{2 N-1}, x\right) Z\left(N ; X_{2 N-1}, y\right)
$$

and

$$
Z_{\mathrm{QT}}\left(4 N+2 ; X_{2 N}, x, y\right)=\sigma(a)^{-1} Z_{\mathrm{HT}}\left(2 N+1 ; X_{2 N}, x, y\right) Z\left(N ; X_{2 N}\right) Z\left(N+1 ; X_{2 N}, x, y\right) \text {. }
$$

Equation (9) is new; equation (8) is due to Kuperberg (4) for the case $x=y$. To see that Theorem 6 implies Theorem 3, we just have to observe that when $a=\omega_{6}$ and all the variables equal to 1 , then the weights at each vertex is $\sigma(a)=\sigma\left(a^{2}\right)$ thus the partition function reduces (up to multiplication by $\left.\sigma(a)^{\text {number of vertices }}\right)$ to the number of states.

\section{Proofs}

In this extended abstract, we shall only give the main ideas of the proofs. Most of them are greatly inspired from (4). To prove Theorem 6, the method is to identify both sides of equations (8) and (9) as Laurent polynomials, and to produce as many specializations of the variables that verify the equalities, as needed to imply these equations in full generality.

\subsection{Laurent polynomials}

Since the weight of any vertex is a Laurent polynomial in the variables $x_{i}, x$ and $y$, the partition functions are Laurent polynomials in these variables. Moreover they are centered Laurent polynomials, i.e. their lowest degree is the negative of their highest degree (called the half-width of the polynomial). In order to divide by two the number of required specializations in $x$, we shall deal with Laurent polynomials of given parity in this variable. To do so, we group together the states with a given orientation (indicated as subscripts in the following notations) at the edge where the parameters $x$ and $y$ meet.

So let us consider the partition functions $Z_{\mathrm{QT}}\left(4 N, X_{2 N-1}, x, y\right)$ and $Z_{\mathrm{QT}}^{\ddagger}\left(4 N, X_{2 N-1}, x, y\right)$, respec-

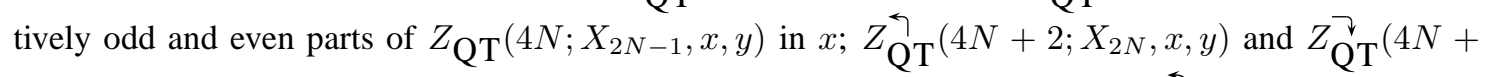
$\left.2 ; X_{2 N}, x, y\right)$, respectively odd and even parts of $Z_{\mathrm{QT}}\left(4 N+2 ; X_{2 N}, x, y\right)$ in $x ; Z_{\mathrm{HT}}^{\Im}\left(2 N ; X_{2 N-1}, x, y\right)$ and $Z_{\mathrm{HT}}^{2}\left(4 N ; X_{2 N-1}, x, y\right)$, respectively parts with the parity of $N$ and of $N-1$ of $Z_{\mathrm{HT}}\left(4 N ; X_{2 N-1}, x, y\right)$ in $x$; and $Z_{\mathrm{HT}^{\top}}^{\top}\left(2 N+1 ; X_{2 N}, x, y\right)$ and $Z_{\mathrm{HT}}^{\nabla}\left(2 N+1 ; X_{2 N}, x, y\right)$, respectively parts with the parity of $N-1$ and of $N$ of $Z_{\mathrm{HT}}\left(2 N+1 ; X_{2 N}, x, y\right)$ in $x$.

With these notations, the equations (8) et (9) are equivalent to the following:

$$
\begin{aligned}
& \sigma(a) Z_{\mathrm{QT}^{\Uparrow}}\left(4 N ; X_{2 N-1}, x, y\right)=Z_{\mathrm{HT}^{\mathrm{T}}}^{\Im}\left(2 N ; X_{2 N-1}, x, y\right) Z\left(N ; X_{2 N-1}, x\right) Z\left(N ; X_{2 N-1}, y\right) \\
& \sigma(a) Z_{\mathrm{QT}}^{\natural}\left(4 N ; X_{2 N-1}, x, y\right)=Z_{\mathrm{HT}}^{\supsetneq}\left(2 N ; X_{2 N-1}, x, y\right) Z\left(N ; X_{2 N-1}, x\right) Z\left(N ; X_{2 N-1}, y\right) \\
& \sigma(a) Z_{\mathrm{QT}}^{\urcorner}\left(4 N+2 ; X_{2 N}, x, y\right)=Z_{\mathrm{HT}}^{\urcorner}\left(2 N+1 ; X_{2 N}, x, y\right) Z\left(N+1 ; X_{2 N}, x, y\right) Z\left(N ; X_{2 N}\right) \\
& \sigma(a) Z_{\mathrm{QT}}\left(4 N+2 ; X_{2 N}, x, y\right)=Z_{\mathrm{HT}}^{\leftarrow}\left(2 N+1 ; X_{2 N}, x, y\right) Z\left(N+1 ; X_{2 N}, x, y\right) Z\left(N ; X_{2 N}\right)
\end{aligned}
$$

Lemma 7 Both left-hand side and right-hand side of equations (10-13) are centered Laurent polynomials in the variable $x$, odd or even, of respective half-widths $2 N-1,2 N-2,2 N$, and $2 N-1$. Thus to prove each of these identities we have to exhibit specializations of $x$ for which the equality is true, and in number strictly exceeding the half-width.

Proof: To compute the half-width of these partition functions, just count the number of vertices in the ice models, and take care that non-zero entries of the ASM (i.e. the first two orientations of Figure 1) give constant weight $\sigma\left(a^{2}\right)$. 


\subsection{Symmetries}

To produce many specializations from one, we shall use symmetry properties of the partition functions. The crucial tool to prove this is the Yang-Baxter equation that we recall below.

Lemma 8 [Yang-Baxter equation] If $x y z=\bar{a}$, then

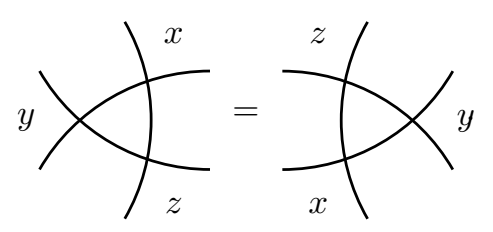

The following lemma gives a (now classical) example of use of the Yang-Baxter equation.

\section{Lemma 9}

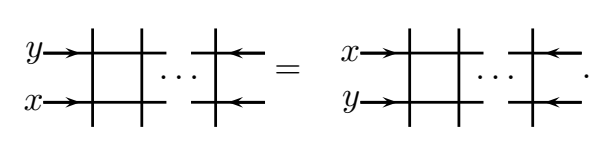

Proof: We multiply the left-hand side by $\sigma(a \bar{z})$, with $z=\bar{a} x \bar{y}$. We get

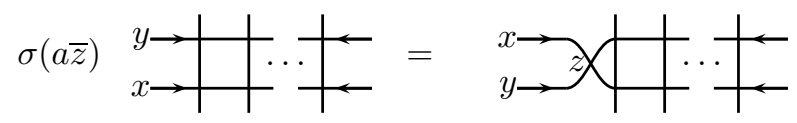

$$
\begin{aligned}
& =x_{y \rightarrow+\infty} \rightarrow+\leftarrow \\
& =\begin{array}{l|l|l|l}
x \rightarrow & & & \ldots 2 \\
y \rightarrow & &
\end{array} \\
& =\begin{array}{l|l|l|l}
x \rightarrow & & \cdots
\end{array} \\
& =\begin{array}{l|l|l|l}
x \rightarrow & & \ldots & \leftarrow \\
y \rightarrow & & & \\
\end{array}
\end{aligned}
$$

The same method, together with the easy transformation

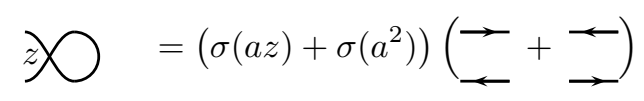

gives the following lemma.

\section{Lemma 10}

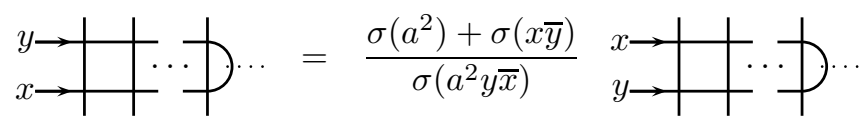

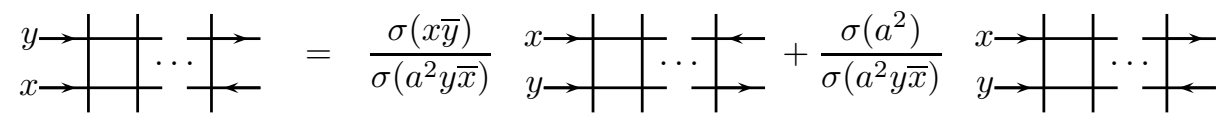

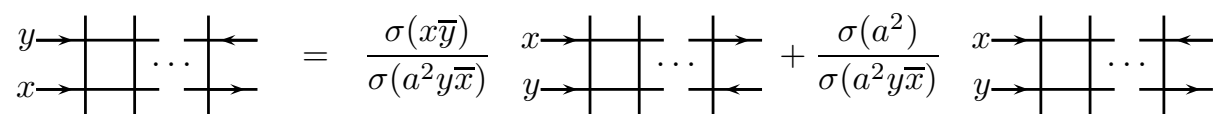

We use Lemmas 9 and 10 to obtain symmetry properties of the partition functions, that we summarize below, where $m$ denotes either $2 N$ or $2 N+1$. 
Lemma 11 The functions $Z\left(N ; X_{2 N}\right)$ and $Z_{\mathrm{HT}}\left(2 N+1 ; X_{2 N}, x, y\right)$ are symmetric separately in the two sets of variables $\left\{x_{i}, i \leq N\right\}$ and $\left\{x_{i}, i \geq N+1\right\}$, the function $Z_{\mathrm{HT}}\left(2 N ; X_{2 N-1}, x, y\right)$ is symmetric separately in the two sets of variables $\left\{x_{i}, i \leq N-1\right\}$ and $\left\{x_{i}, i \geq N\right\}$, and the functions $Z_{\mathrm{QT}}\left(2 m ; X_{N-1}, x, y\right)$ are symmetric in their variables $x_{i}$.

Moreover, $Z_{\mathrm{QT}}(4 N+2 ; \ldots)$ is symmetric in its variables $x$ and $y$, and we have a pseudo-symmetry for $Z_{\mathrm{QT}}(4 N ; \ldots)$ and $Z_{\mathrm{HT}}(2 N ; \ldots)$ :

$$
\begin{aligned}
Z_{\mathrm{QT}}\left(4 N ; X_{2 N-1}, x, y\right) & =\frac{\sigma\left(a^{2}\right)+\sigma(x \bar{y})}{\sigma\left(a^{2} y \bar{x}\right)} Z_{\mathrm{QT}}\left(4 N ; X_{2 N-1}, y, x\right) \\
Z_{\mathrm{HT}}\left(2 N ; X_{2 N-1}, x, y\right) & =\frac{\sigma\left(a^{2}\right)+\sigma(x \bar{y})}{\sigma\left(a^{2} y \bar{x}\right)} Z_{\mathrm{HT}}\left(2 N ; X_{2 N-1}, y, x\right) .
\end{aligned}
$$

Proof: For $Z(N ; \ldots)$ and $Z_{\mathrm{HT}}(m ; \ldots)$, the symmetry in two "consecutive" variables $x_{i}$ and $x_{i+1}$ is a direct consequence of Lemma 9 . For $Z_{\mathrm{QT}}(2 m ; \ldots)$, we again apply Lemma 9 together with the easy observations:

$$
+=\rightarrow \uparrow^{\prime} \text { and } \longrightarrow=\ldots
$$

which allow us to bring the Yang-Baxter triangle through the dotted lines of Figure 4.

For the (pseudo-)symmetries in $(x, y)$, let us deal with $Z_{\mathrm{QT}}(4 N ; \ldots)$, the other cases being similar or simpler. We use equation (22) to put together the lines of parameter $x$ and $y$ :

and then apply Lemma 10.

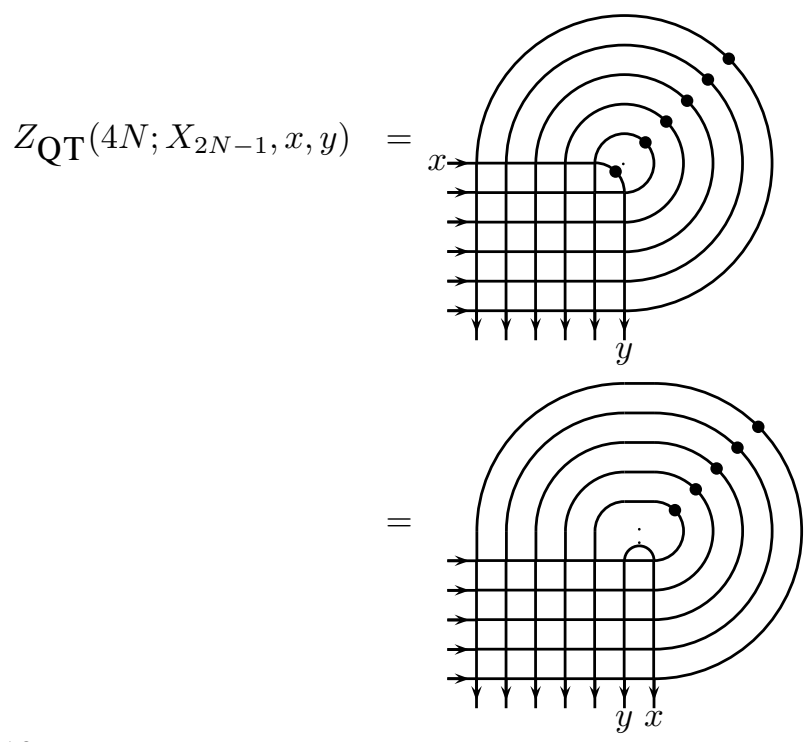

It should be clear that we have analogous properties for the even and odd parts of the partition functions. The next (and last) symmetry property was proved by Stroganov (8) (a recent and elementary proof may be found in (1)). It appears when the parameter $a$ equals the special value $\omega_{6}=\exp (i \pi / 3)$.

Lemma 12 When $a=\omega_{6}$, the partition function $Z\left(N ; X_{2 N}\right)$ is symmetric in all its variables.

\subsection{Specializations, recurrences}

The aim of this section is to give the value of the partition functions in some specializations of the variable $x$ or $y$. The first result is due to Kuperberg, the other are very similar. 
Lemma 13 [specialization of $Z$; Kuperberg] If we denote

$$
\begin{aligned}
& A\left(x_{N+1}, X_{2 N} \backslash\left\{x_{1}, x_{N+1}\right\}\right)=\prod_{2 \leq k \leq N} \sigma\left(a x_{k} \bar{x}_{N+1}\right) \prod_{N+1 \leq k \leq 2 N} \sigma\left(a^{2} x_{N+1} \bar{x}_{k}\right), \\
& \bar{A}\left(x_{N+1}, X_{2 N} \backslash\left\{x_{1}, x_{N+1}\right\}\right)=\prod_{2 \leq k \leq N} \sigma\left(a x_{N+1} \bar{x}_{k}\right) \prod_{N+1 \leq k \leq 2 N} \sigma\left(a^{2} x_{k} \bar{x}_{N+1}\right),
\end{aligned}
$$

then we have:

$$
\begin{aligned}
& Z\left(N ; \overline{\mathbf{a}} \mathbf{x}_{\mathbf{N}+\mathbf{1}}, X_{2 N} \backslash x_{1}\right)=\bar{A}\left(x_{N+1}, X_{2 N} \backslash\left\{x_{1}, x_{N+1}\right\}\right) Z\left(N-1 ; X_{2 N} \backslash\left\{x_{1}, x_{N+1}\right\}\right) \\
& Z\left(N ; \mathbf{a x}_{\mathbf{N}+\mathbf{1}}, X_{2 N} \backslash x_{1}\right)=A\left(x_{N+1}, X_{2 N} \backslash\left\{x_{1}, x_{N+1}\right\}\right) Z\left(N-1 ; X_{2 N} \backslash\left\{x_{1}, x_{N+1}\right\}\right) .
\end{aligned}
$$

Proof: We recall the method to prove equation (23). We observe that when $x_{1}=\bar{a} x_{N+1}$, the parameter of the vertex at the crossing of the two lines of parameter $x_{1}$ and $x_{N+1}$ is $\bar{a}$. Thus the weight of this vertex is $\sigma(a \bar{a})=\sigma(1)=0$ unless the orientation of this vertex is the second on Figure 1. But this orientation implies the orientation of all vertices in the row $x_{N+1}$ and in the column $x_{1}$, as shown on Figure 5 . The fixed part gives the partition function $Z$ in size $N-1$, without parameters $x_{1}$ and $x_{N+1}$, and the weights of the fixed part gives the factor $\bar{A}(\ldots)$.
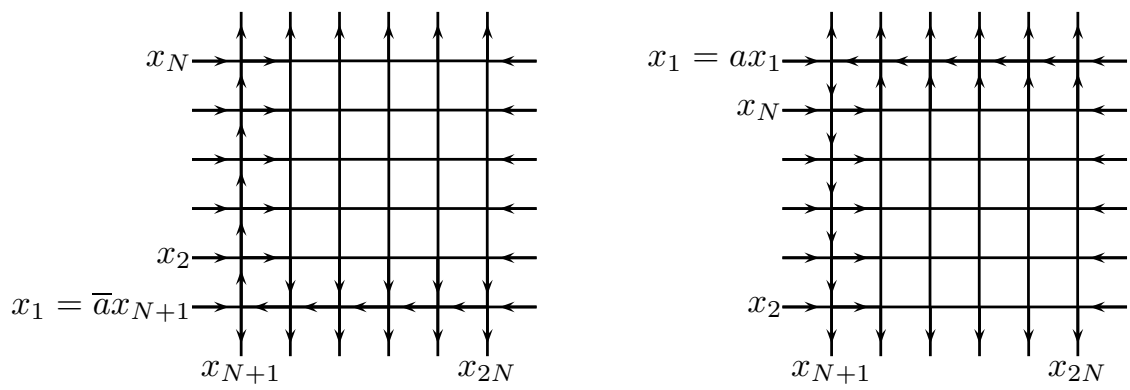

Fig. 5: Fixed edges for (23) on the left and (24) on the right

The case of (24) is similar, after using Lemma 11 to put the line $x_{N+1}$ at the top of the grid.

We will need the following application of the Yang-Baxter equation, which allows, under certain condition, a line with a change of parameter to go through a grid.

\section{Lemma 14}

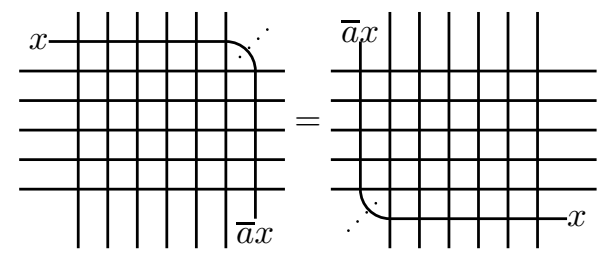

Proof: We iteratively apply Lemma 8 on the rows, and row by row:

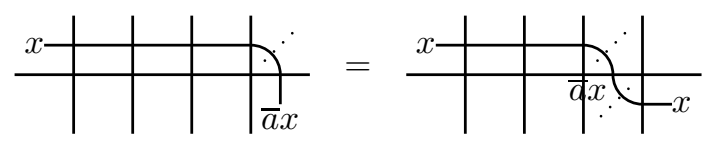




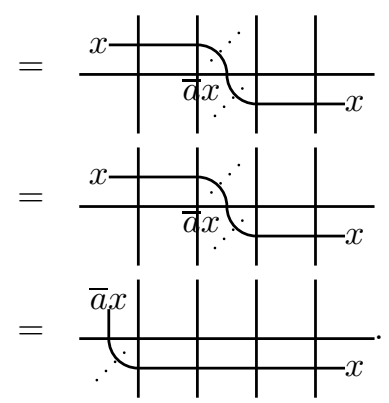

Lemma 15 [specialization of $Z_{\mathrm{HT}}$ ] If we denote

$$
\begin{aligned}
A_{H}^{1}\left(x_{1}, X_{2 N} \backslash x_{1}\right) & =\prod_{1 \leq k \leq N} \sigma\left(a^{2} x_{1} \bar{x}_{k}\right) \prod_{N+1 \leq k \leq 2 N} \sigma\left(a x_{k} \bar{x}_{1}\right) \\
\bar{A}_{H}^{1}\left(x_{1}, X_{2 N} \backslash x_{1}\right) & =\prod_{1 \leq k \leq N} \sigma\left(a^{2} x_{k} \bar{x}_{1}\right) \prod_{N+1 \leq k \leq 2 N} \sigma\left(a x_{1} \bar{x}_{k}\right) \\
A_{H}^{0}\left(x_{N}, X_{2 N-1} \backslash x_{N}\right) & =\prod_{1 \leq k \leq N-1} \sigma\left(a x_{k} \bar{x}_{N}\right) \prod_{N \leq k \leq 2 N-1} \sigma\left(a^{2} x_{N} \bar{x}_{k}\right) \\
\bar{A}_{H}^{0}\left(x_{N}, X_{2 N-1} \backslash x_{N}\right) & =\prod_{1 \leq k \leq N-1} \sigma\left(a x_{N} \bar{x}_{k}\right) \prod_{N \leq k \leq 2 N-1} \sigma\left(a^{2} x_{k} \bar{x}_{N}\right),
\end{aligned}
$$

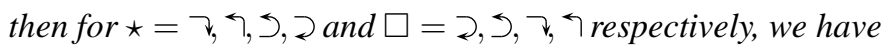

$$
\begin{aligned}
Z_{\mathrm{HT}}^{\star}\left(2 N+1 ; X_{2 N}, x, \mathbf{a x}\right. & =A_{H}^{1}\left(x_{1}, X_{2 N} \backslash x_{1}\right) Z_{\mathrm{HT}}^{\square}\left(2 N ; X_{2 N} \backslash x_{1}, x_{1}, x\right) \\
Z_{\mathrm{HT}}^{\square}\left(2 N+1 ; X_{2 N}, x, \overline{\mathbf{a}} \mathbf{x}_{\mathbf{1}}\right) & =\bar{A}_{H}^{1}\left(x_{1}, X_{2 N} \backslash x_{1}\right) Z_{\mathrm{HT}}^{\star}\left(2 N ; X_{2 N} \backslash x_{1}, x, x_{1}\right) \\
Z_{\mathrm{HT}}^{\star}\left(2 N ; X_{2 N-1}, x, \mathbf{a x}_{\mathbf{N}}\right) & =\sigma\left(a x \bar{x}_{N}\right) A_{H}^{0}\left(x_{N}, X_{2 N-1} \backslash x_{N}\right) Z_{\mathrm{HT}}^{\square}\left(2 N-1 ; X_{2 N-1} \backslash x_{N}, x, x_{N}\right) \\
Z_{\mathrm{HT}}^{\square}\left(2 N ; X_{2 N-1}, \overline{\mathbf{a}} \mathbf{x}_{\mathbf{N}}, y\right) & =\sigma\left(a x_{N} \bar{y}\right) \bar{A}_{H}^{0}\left(x_{N}, X_{2 N-1} \backslash x_{N}\right) Z_{\mathrm{HT}}^{\star}\left(2 N-1 ; X_{2 N-1} \backslash x_{N}, y, x_{N}\right)
\end{aligned}
$$

\section{Proof:}

The proof is similar to the previous one, with the difference that before looking at fixed edges, we need to multiply the partition function by a given factor; we interpret this operation by a modification of the graph of the ice model and apply Lemma 14. It turns out that in each case, the additional factors are exactly cancelled by the weights of fixed vertices.

To prove (26), we multiply the left-hand side by

$$
\prod_{N+1 \leq k \leq 2 N} \sigma\left(a^{2} x_{k} \bar{y}\right)
$$

which is equivalent to adding to the line of parameter $y$ a new line $\bar{a} y$ just below the grid; the Lemma 14 transforms the graph of Figure 6(a) into the graph of Figure 6(b). When we put $y=a x_{1}$,we get the indicated fixed edges, which gives as partition function

$$
\prod_{N+1 \leq k \leq 2 N} \sigma^{2}\left(a x_{k} \bar{x}_{1}\right) \prod_{1 \leq k \leq N} \sigma\left(a^{2} x_{1} \bar{x}_{k}\right) Z_{\mathrm{HT}}\left(2 N ; X_{2 N} \backslash x_{1}, x_{1}, x\right) .
$$

Since $a^{2} x_{k} \bar{y}=a x_{k} \bar{x}_{1}$, the equation simplifies. Ton conclude, we observe that if we start with an edge going out from the crossing $x / x_{2 N}$ (function $Z_{\mathrm{HT}}^{\supsetneq}$ ) we get at the end the same orientation (function $Z_{\mathrm{HT}}^{\mathrm{T}}$ ). 


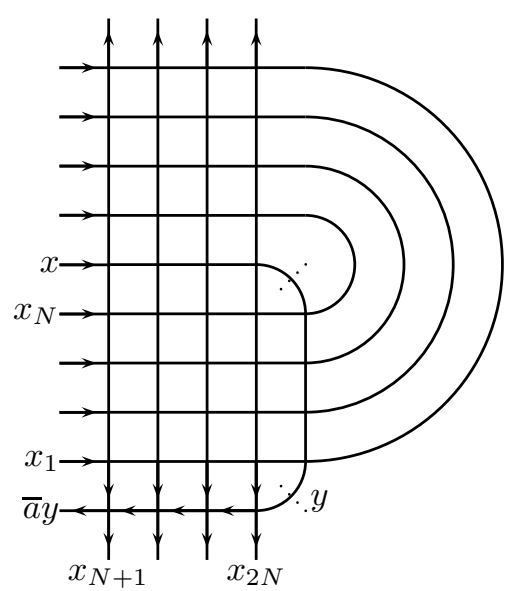

(a)

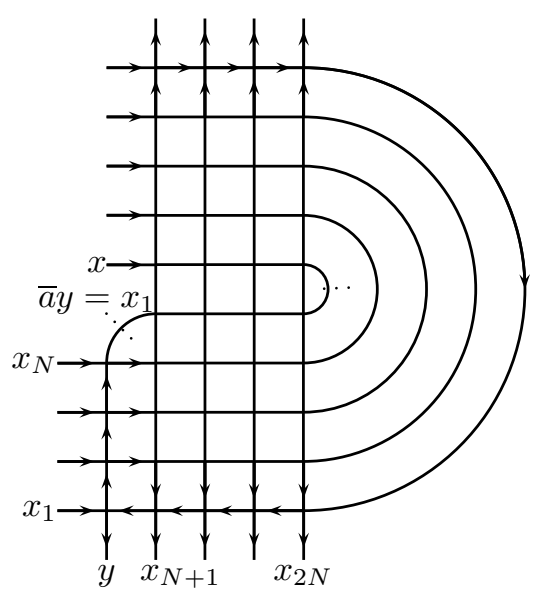

(b)

Fig. 6: Proof of (26)

Lemma 16 [specialization of $Z_{\mathrm{QT}}$ ] If we denote

$$
\begin{aligned}
& \bar{A}_{Q}\left(x_{1}, X_{m-1} \backslash x_{1}\right)=\prod_{1 \leq k \leq m-1} \sigma\left(a^{2} x_{k} \bar{x}_{1}\right) \sigma\left(a x_{1} \bar{x}_{k}\right) \\
& A_{Q}\left(x_{1} ; X_{m-1} \backslash x_{1}\right)=\prod_{1 \leq k \leq m-1} \sigma\left(a^{2} x_{1} \bar{x}_{k}\right) \sigma\left(a x_{k} \bar{x}_{1}\right)
\end{aligned}
$$

then for $\star=\rightarrow, ~ \neg,\urcorner, \uparrow$ and $\square=\uparrow,\urcorner, \rightarrow, \neg$ respectively, we have:

$$
\begin{aligned}
& Z_{\mathrm{QT}}^{\star}\left(2 m ; X_{m-1}, \overline{\mathbf{a}} \mathbf{x}_{\mathbf{1}}, y\right)=\sigma\left(a x_{1} \bar{y}\right) \bar{A}_{Q}\left(x_{1}, X_{m-1}\right) Z_{\mathrm{QT}}^{\square}\left(2 m-2 ; X_{m-1} \backslash x_{1}, y, x_{1}\right) \\
& Z_{\mathrm{QT}}^{\square}\left(2 m ; X_{m-1}, x, \mathbf{a x}_{\mathbf{1}}\right)=\sigma\left(a x \bar{x}_{1}\right) A_{Q}\left(x_{1} ; X_{m-1} \backslash x_{1}\right) Z_{\mathrm{QT}}^{\star}\left(2 m-2 ; X_{m-1} \backslash x_{1}, x_{1}, x\right)
\end{aligned}
$$

Proof: Similar to the proof of Lemma 15.

Remark 17 By using the (pseudo-)symmetry in ( $x, y$ ), we may transform any specialization of the variable $y$ into a specialization of the variable $x$. Moreover, by using Lemma 11 and (when $a=\omega_{6}$ ) Lemma 12, we obtain for $Z, Z_{\mathrm{HT}}$ and $Z_{\mathrm{QT}} Z 2 N$ specializationse now have to compare them.

\subsection{Special value of the parameter $a$; conclusion}

When $a=\omega_{6}=\exp (i \pi / 3)$, two new ingredients may be used. The first one is Lemma 12, as mentionned in Remark 17. The second one is that with this special value of $a$ :

$$
\sigma(a)=\sigma\left(a^{2}\right) \quad \sigma\left(a^{2} x\right)=-\sigma(\bar{a} x)=\sigma(a \bar{x}) .
$$

which implies that the products appearing in Lemmas 13, 15 and 16 may be written in a more compact way:

$$
\begin{aligned}
A\left(x_{N+1}, X_{2 N} \backslash\left\{x_{1}, x_{N+1}\right\}\right) & =\sigma(a) \prod_{k \neq 1, N+1} \sigma\left(a x_{k} \bar{x}_{N+1}\right), \\
\bar{A}\left(x_{N+1}, X_{2 N} \backslash\left\{x_{1}, x_{N+1}\right\}\right) & =\sigma(a) \prod_{k \neq 1, N+1} \sigma\left(a x_{N+1} \bar{x}_{k}\right), \\
A_{H}^{1}\left(x_{1}, X_{2 N} \backslash x_{1}\right) & =\prod_{1 \leq k \leq 2 N} \sigma\left(a x_{k} \bar{x}_{1}\right),
\end{aligned}
$$


Enumeration of alternating sign matrices of even size (quasi)-invariant under a quarter-turn rotation 125

$$
\begin{aligned}
\bar{A}_{H}^{1}\left(x_{1}, X_{2 N} \backslash x_{1}\right) & =\prod_{1 \leq k \leq 2 N} \sigma\left(a x_{1} \bar{x}_{k}\right), \\
A_{H}^{0}\left(x_{N}, X_{2 N-1} \backslash x_{N}\right) & =\prod_{1 \leq k \leq 2 N-1} \sigma\left(a x_{k} \bar{x}_{N}\right) \\
\bar{A}_{H}^{0}\left(x_{N}, X_{2 N-1} \backslash x_{N}\right) & =\prod_{1 \leq k \leq 2 N-1} \sigma\left(a x_{N} \bar{x}_{k}\right), \\
\bar{A}_{Q}\left(x_{1}, X_{m-1} \backslash x_{1}\right) & =\prod_{1 \leq k \leq m-1} \sigma^{2}\left(a x_{1} \bar{x}_{k}\right), \\
A_{Q}\left(x_{1}, X_{m-1} \backslash x_{1}\right) & =\prod_{1 \leq k \leq m-1} \sigma^{2}\left(a x_{k} \bar{x}_{1}\right) .
\end{aligned}
$$

Thus we get by comparing:

$$
\begin{aligned}
A\left(x_{i}, X_{2 N} \backslash x_{i}, x\right) A_{H}^{1}\left(x_{i}, X_{2 N} \backslash x_{i}\right) & =\sigma\left(a x \bar{x}_{i}\right) A_{Q}\left(x_{i}, X_{2 N} \backslash x_{i}\right) \\
\bar{A}\left(x_{i}, X_{2 N} \backslash x_{i}, x\right) \bar{A}_{H}^{1}\left(x_{i}, X_{2 N} \backslash x_{i}\right) & =\sigma\left(a x_{i} \bar{x}\right) \bar{A}_{Q}\left(x_{i}, X_{2 N} \backslash x_{i}\right),
\end{aligned}
$$

whence (10) and (11) imply that (12) and (13) are true (in size $4 N+2$ ) for the $2 N$ specializations $y=$ $a^{(+,-)} x_{i}(1 \leq i \leq N)$. It is enough to prove (13) (Laurent polynomials of half-width $2 N-1$ ), but we still need one specialization to get (12) (half-width $2 N$ ).

For (10) and (11), we observe the same kind of simplification

$$
A\left(x_{i}, X_{2 N-1} \backslash x_{i}\right) \sigma\left(a x \bar{x}_{i}\right) A_{H}^{0}\left(x_{i}, X_{2 N-1} \backslash x_{i}\right)=\sigma\left(a x \bar{x}_{i}\right) A_{Q}\left(x_{i}, X_{2 N-1} \backslash x_{i}\right),
$$

whence (13) and (12) for the size $4 N-2$ imply that (10) and (11) are true for the $N$ specializations $x=a x_{i}, N \leq i \leq 2 N-1$. We obtain in the same way the coincidence for the $N$ specializations $x=\bar{a} x_{i}$, $N \leq i \leq 2 N-1$. Thus we have $2 N$ specialiations of $x$ : it is enough both for (10) (half-width $2 N-1$ ), and for (11) (half-width $2 N-2$ ).

At this point, we have almost proved

$$
((10) \text { and (11), size } 4 N) \Longrightarrow(((12) \text { and (13), size } 4 N+2) \Longrightarrow((10) \text { and (11), size } 4 N+4) \text {; }
$$

almost, because we still need one specialization for (12).

We get this missing speciazation, not directly for $Z_{\mathrm{QT}}^{\nabla}, Z_{\mathrm{QT}}^{\curlyvee}, Z_{\mathrm{HT}}^{\curvearrowright}$ and $Z_{\mathrm{HT}}^{\curlyvee}$, but for the original series $Z_{\mathrm{QT}}\left(4 N+2 ; X_{2 N}, x, y\right)$ and $Z_{\mathrm{HT}}\left(2 N+1 ; X_{2 N}, x, y\right)$ : indeed if we set $x=a y$ we may apply Lemma 14.
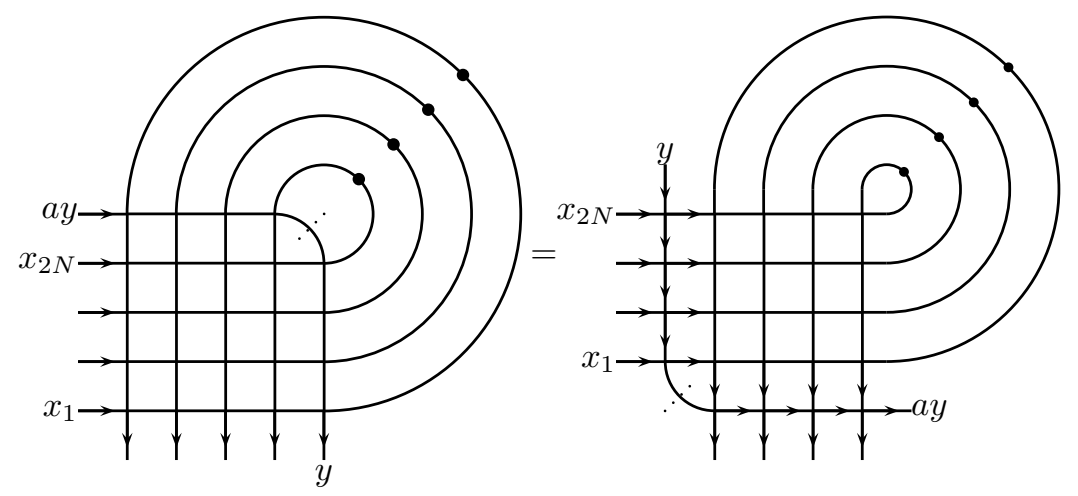

$$
Z_{\mathrm{QT}}\left(4 N+2 ; X_{2 N}, \text { ay }, y\right)=\sigma(a) \prod_{1 \leq k \leq 2 N} \sigma\left(a x_{k} \bar{y}\right) \sigma\left(a^{2} y \bar{x}_{k}\right) Z_{\mathrm{QT}}\left(4 N ; X_{2 N} \backslash x_{2 N}, x_{2 N}, x_{2 N}\right)
$$



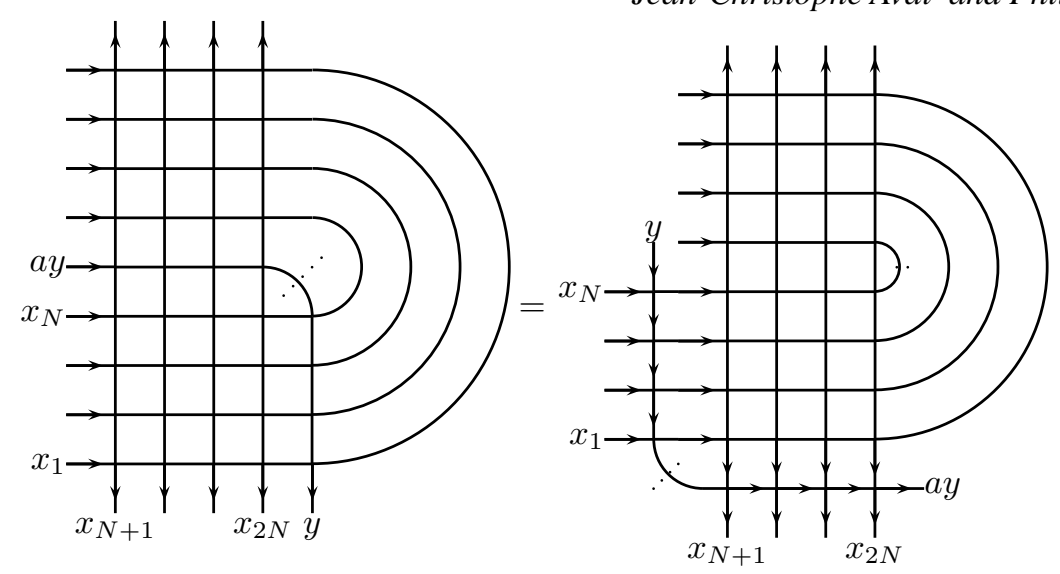

$Z_{\mathrm{HT}}\left(2 N+1 ; X_{2 N}, a y, y\right)=\left(\prod_{1 \leq k \leq N} \sigma\left(a x_{k} \bar{y}\right) \prod_{N+1 \leq k \leq 2 N} \sigma\left(a^{2} y \bar{x}_{k}\right)\right) Z_{\mathrm{HT}}\left(2 N ; X_{2 N} \backslash x_{N}, x_{N}, x_{N}\right)$

This way, we get another point where (9) is true, and thus, because we already have (13), by difference we obtain that (12) holds for $y=\bar{a} x$.

This completes the proof of Theorem 6 .

\section{References}

[1] J.-C. AvaL, On the symmetry of the partition function of some square ice models, to appear in Theor. Math. Phys, arXiv:0903.0777.

[2] P. DuCHON, On the link pattern distribution of quarter-turn symmetric FPL configurations, FPSAC 2008, Valparaiso (Chile), DMTCS proceedings, 331-342.

[3] G. KUPERBERG, Another proof of the alternating sign matrices conjecture, Internat. Math. Research Not. ,1996 (1996), 139-150.

[4] G. KUPerberg, Symmetry classes of alternating sign matrices under one roof, Ann. Math. 156 (2002) 835-866.

[5] W. Mills, D. Robbins, H. Rumsey, Alternating sign matrices and descending plane partitions, J. Combin. Th. Ser. A 34 (1983), 340-359.

[6] A. V. RAzUmov, Y. G. StRoganov, Enumeration of quarter-turn symmetric alternating sign matrices of odd order, Theoret. Math. Phys. 149 (2006) 1639-1650.

[7] D. RobBINS, Symmetry classes of alternating sign matrices, arXiv : math. CO/0008045.

[8] Y. G. Stroganov, A new way to deal with Izergin-Korepin determinant at root of unity, arXiv:math-ph/0204042.

[9] D. Zeilberger, Proof of the alternating sign matrix conjecture, Electronic J. Combinatorics 3 , No. 2 (1996), R13, 1-84. 